\title{
Synthesis of Hexahydropyrazino[1,2-b]isoquinolines as Simplified Saframycin Analogues
}

\author{
Tuyen Nguyen Van, ${ }^{1}$ Pieter Claes, Norbert De Kimpe* \\ Department of Sustainable Organic Chemistry and Technology, Faculty of Bioscience Engineering, Ghent University, Coupure Links 653, \\ 9000 Ghent, Belgium \\ Fax +32(9)26462 21; E-mail: norbert.dekimpe@ugent.be \\ Received: 12.07.2013; Accepted after revision: 01.10.2013
}

\begin{abstract}
Various hexahydropyrazino[1,2-b]isoquinolines were synthesised as simplified saframycin analogues. Construction of this core proceeded through a tetrahydroisoquinoline synthesis followed by acylation/alkylation of the tetrahydroisoquinoline nitrogen and subsequent ring closure using various aliphatic and aromatic amines. The resulting piperazinones were reacted with $\mathrm{LiAlH}_{4}$ or $\mathrm{LiAlH}(\mathrm{OEt})_{3}$ to synthesise further analogues.
\end{abstract}

Key words: saframycins, piperazinones, diketopiperazines, cyclisation, tetrahydroisoquinolines

Saframycins 1, isolated from Streptomyces lavendulae, belong to a family of microbial fermentation products with a remarkable antiproliferative activity. The most active derivative is saframycin A (1a), a bisquinone alkaloid bearing an $\alpha$-aminonitrile function. ${ }^{2}$ The mode of activity is connected to the iminium ions generated from this $\alpha$-aminonitrile unit thus covalently modifying DNA. Quinoid alkaloids with antiproliferative activity such as the ecteinascidines, ${ }^{3}$ isolated from the marine tunicate Ecteinascidia turbinata, have raised new interest towards the synthesis of saframycin analogues.

Trabectedin (2; also known as ecteinascidin 743 or ET-743) is an antitumor drug approved for the treatment of advanced soft-tissue sarcoma. It is sold by Zeltia and Johnson \& Johnson under the brand name Yondelis for the treatment of advanced soft-tissue sarcoma. Currently, simplified analogues such as phthalascidin (3) are known, bearing a similar activity ${ }^{4}$ (Figure 1).

All these compounds can be considered as dimers of structurally less complex tetrahydroisoquinoline subunits. Synthesis of this kind of simplified analogues has received little attention as most work focusses on total synthesis. ${ }^{5}$ Nevertheless, related piperazinones and diketopiperazines have been prepared before ${ }^{6}$ and pyrazino[1,2$b]$ isoquinolines have been examined for cytotoxicity. Therefore, the synthesis of quinone-type derivatives under their hydroquinone methyl ether form was envisaged.

We recently reported the synthesis of functionalised diketopiperazines as cyclotryprostatin and tryprostatin analogues. ${ }^{8}$ It was subsequently envisaged to apply this methodlogy to the synthesis of simplified saframycin

SYNLETT 2014, 25, 0069-0074

Advanced online publication: 13.11.2013

DOI: 10.1055/s-0033-1340070; Art ID: ST-2013-D0646-L

C Georg Thieme Verlag Stuttgart · New York analogues. In initial studies, ethyl $N$-(diphenylmethylene)glycinate (4) failed to react with bromomethyl derivatives 5 (Na or KHMDS, $-78{ }^{\circ} \mathrm{C}$ or $0{ }^{\circ} \mathrm{C}^{9}$ ) but complete conversion was obtained upon reaction with $\mathrm{KOH}$ in $\mathrm{H}_{2} \mathrm{O}-\mathrm{CH}_{2} \mathrm{Cl}_{2}$ using $\mathrm{Bu}_{4} \mathrm{NHSO}_{4}$ as a phase-transfer catalyst. Tetrahydroisoquinoline 7a was synthesised by means of a Pictet-Spengler reaction starting from 1-bromomethyl-2,5-dimethoxy-3,4-dimethylbenzene (5a) ${ }^{10 a}$ via intermediate amine 6 in a yield of $76 \%$ over two steps. Tetrahydroisoquinolines $\mathbf{7 b}-\mathbf{d}$ were synthesised by reaction of bis(bromomethyl)benzene derivatives $5 \mathbf{b}-\mathbf{d}^{10 \mathrm{~b}-\mathrm{e}}$ with ethyl $N$-(diphenylmethylene)glycinate (4) under basic conditions followed by acid-induced ring closure in

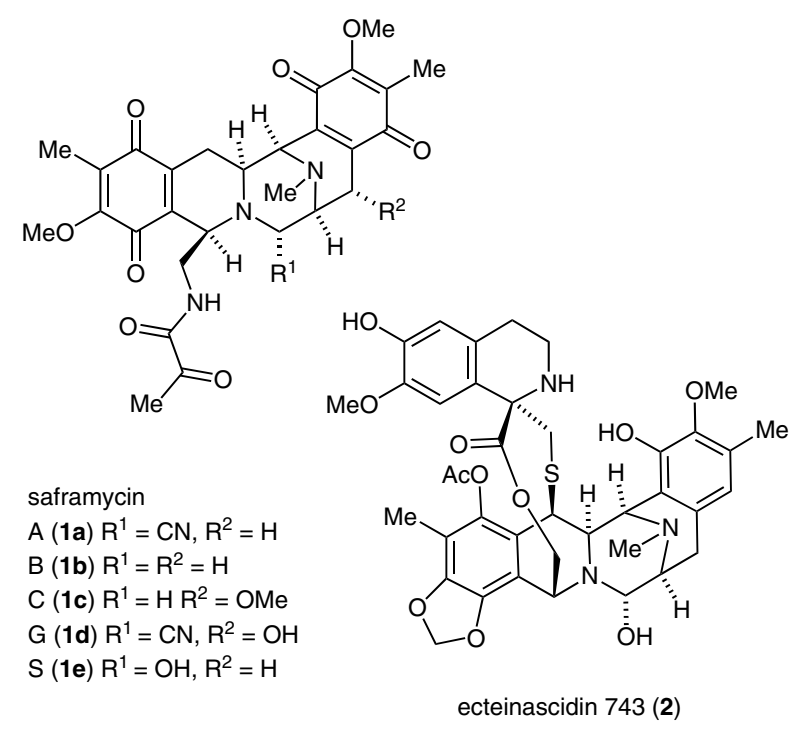

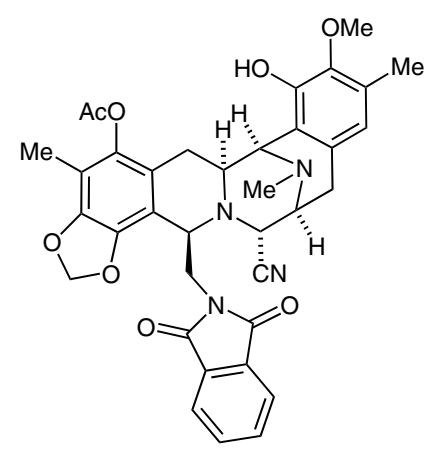

phthalascidin (3)

Figure 1 Examples of saframycins 1, trabectedin (ecteinascidin 743, 2), and the structurally related phthalascidin (3) 
$60-80 \%$ yields. ${ }^{11}$ Next, the nitrogen atom was acylated with chloroacetyl chloride to afford $N$-chloroacetyl tetrahydroisoquinolines 8 in $50-70 \%$ yield $^{12}$ or alkylated with 1,2-dibromoethane to yield $N$-(2-bromoethyl)tetrahydroisoquinolines 9 in $70-94 \%$ yield $^{13}$ (Scheme 1). Finally, $N$-chloroacetyl tetrahydroisoquinolines $\mathbf{8}$ were reacted with various primary amines in EtOH towards diketopiperazines 10 in good to excellent yields (Table 1). ${ }^{14}$<smiles>CCOC(=O)CC(N)Cc1cc(OC)c(C)c(C)c1OC</smiles>$$
4
$$$$
5 a
$$

$6(92 \%)$<smiles>CCOC(=O)CN=C(c1ccccc1)c1ccccc1</smiles><smiles>COc1ccc(OC)c(CBr)c1CBr</smiles><smiles>[1H]c1c([14CH3])c(OC)c2c(c1OC)CNC(C(=O)OCC)C2</smiles>

4 $5 b \mathrm{R}^{1}=\mathrm{H}$ $5 c \mathrm{R}^{1}=\mathrm{Br}$

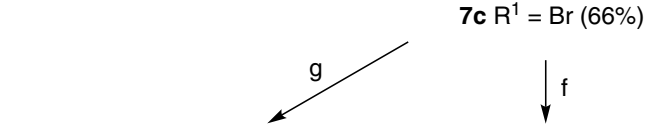<smiles>CCOC(=O)C1Cc2c(OC)cc(OC)c(OC)c2CN1CCBr</smiles><smiles></smiles>

9a $\mathrm{R}^{1}=\mathrm{Me}(54 \%)$

9b $\mathrm{R}^{1}=\mathrm{H}(70 \%)$

$8 \mathbf{a} R^{1}=\mathrm{Me}(85 \%)$

$8 b R^{1}=H(93 \%)$

8c $\mathrm{R}^{1}=\mathrm{Br}(94 \%)$<smiles>CCOC(=O)CN=C(c1ccccc1)c1ccccc1</smiles><smiles>CCOC(=O)C1Cc2c(c(OC)c3ccccc3c2OC)CN1</smiles><smiles>CCOC(=O)C1Cc2c(c(OC)c3ccccc3c2OC)CN1CCBr</smiles>

9d (50\%)<smiles>CCOC(=O)C1Cc2c(c(OCC)c3ccccc3c2O[14C](C)(C)C)CN1C(=O)CCl</smiles>

Scheme 1 Reagents and conditions: a) $30 \%$ aq $\mathrm{KOH}, \mathrm{Bu}_{4} \mathrm{NHSO}_{4}(1$ equiv), $\mathrm{CH}_{2} \mathrm{Cl}_{2}$, r.t., $12 \mathrm{~h}$; b) $\mathrm{HCl}(2 \mathrm{M}) \mathrm{THF}$, r.t., 15 h; c) $37 \% \mathrm{HCHO}$ in $\mathrm{H}_{2} \mathrm{O}$ (2 equiv), TFA (2 equiv), $\mathrm{CH}_{2} \mathrm{Cl}_{2}, \Delta, 2 \mathrm{~h}$; d) $30 \%$ aq $\mathrm{KOH}$, $\mathrm{Bu}_{4} \mathrm{NHSO}_{4}$ (1 equiv), $\mathrm{CH}_{2} \mathrm{Cl}_{2}$, r.t., 30 min; e) $\mathrm{HCl}(2 \mathrm{M})$ THF, r.t., 30 min; f) $\mathrm{ClCH}_{2} \mathrm{COCl}$ (1.5 equiv), $\mathrm{Et}_{3} \mathrm{~N}$ (1.5 equiv), $\mathrm{CH}_{2} \mathrm{Cl}_{2}$, r.t., $2 \mathrm{~h} ; \mathrm{g}$ ) $\mathrm{BrCH}_{2} \mathrm{CH}_{2} \mathrm{Br}$ (20 equiv), $\mathrm{K}_{2} \mathrm{CO}_{3}$ (1 equiv), neat, $80^{\circ} \mathrm{C}, 24 \mathrm{~h}$.
Starting from $N$-(2-bromoethyl)tetrahydroisoquinolines $\mathbf{9}$, a range of piperazinones $\mathbf{1 1}$ was synthesised in high, albeit somewhat lower yields than diketopiperazines $\mathbf{1 0}$ (Table 2). ${ }^{15}$

The lactam function of piperazinones $\mathbf{1 1}$ was further reduced to create additional saframycin analogues. Reaction with $\mathrm{LiAlH}_{4}$ resulted in complete reduction of the lactam moiety leading to piperazines 14 in $70-80 \%$ yield. ${ }^{16}$ Reaction with the less reactive $\mathrm{LiAlH}(\mathrm{OEt})_{3}$ gave the hemiaminals, which were further converted into aminonitriles 13 with potassium cyanide and acetic acid. ${ }^{17}$ One piperazinone was demethylated with boron(III) bromide followed by oxidation with $\mathrm{HNO}_{3}$ to yield quinone $\mathbf{1 2}$ (Scheme 2). ${ }^{18}$<smiles>[R]N1CCN2CC3=C(CC2C1=O)C(=O)C=CC3=O</smiles>
$12 \mathrm{R}^{2}=\mathrm{Bn}(68 \%)$<smiles>CCCN1CCN2Cc3c(OC)ccc(OC)c3CC2C1=O</smiles><smiles>[12H][131IH]</smiles>
14a $\mathrm{R}^{2}=n-\operatorname{Pr}(80 \%)$<smiles>[R]N1CCN2Cc3c(OC)ccc(OC)c3CC2(C)C1</smiles>

13a $\mathrm{R}^{2}=n-\operatorname{Pr}(84 \%)$ 13b $\mathrm{R}^{2}=\mathrm{Ph}\left(\mathrm{CH}_{2}\right)_{2}(85 \%)$ $13 \mathrm{c} \mathrm{R}^{2}=4-\mathrm{ClC}_{6} \mathrm{H}_{4}\left(\mathrm{CH}_{2}\right)_{2}(79 \%)$<smiles>CCCN1CCN2Cc3c(c(OC)c4ccccc4c3OC)CC3C(=O)N(P)CCN3CC2C1</smiles><smiles>COc1c2c(c(OC)c3ccccc13)CC1(C)CN([Tl])CCN1C2</smiles>

13d $R^{2}=n-\operatorname{Pr}(78 \%)$ 13e $\mathrm{R}^{2}=4-\mathrm{ClC}_{6} \mathrm{H}_{4}\left(\mathrm{CH}_{2}\right)_{2}(85 \%)$

Scheme 2 Reagents and conditions: a) $\mathrm{BBr}_{3}\left(2.1\right.$ equiv), $-78^{\circ} \mathrm{C}, 1 \mathrm{~h}$, then $0{ }^{\circ} \mathrm{C}, 45 \mathrm{~min}$; b) $\mathrm{HNO}_{3}(10 \mathrm{M})$, r.t., $45 \mathrm{~min}$; c) $\mathrm{LiAlH}_{4}$ (4 equiv), $\mathrm{Et}_{2} \mathrm{O}$, r.t., $4 \mathrm{~h}$; d) $\mathrm{LiAlH}(\mathrm{OEt})_{3}$ (10 equiv), THF, $0{ }^{\circ} \mathrm{C}, 30 \mathrm{~min}$; e) $\mathrm{AcOH}$ (40 equiv), $\mathrm{KCN}$ (6 equiv), $\mathrm{H}_{2} \mathrm{O}$, r.t., 3 h. 
Table 1 Synthesis of Diketopiperazines 10

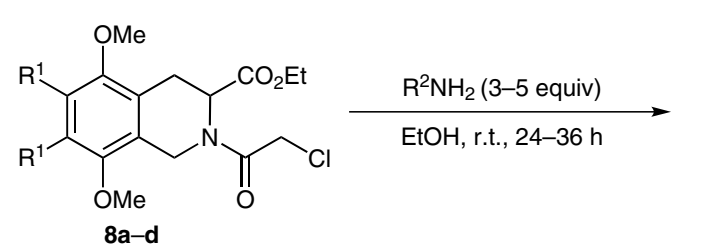<smiles>[R]c1c([R])c(OC)c2c(c1OC)CC1C(=O)N(CC)CC(=O)N1C2</smiles>

\begin{tabular}{|c|c|c|c|c|}
\hline Compd & $\mathrm{R}^{1}, \mathrm{R}^{1}$ & $\mathrm{R}^{2}$ & Time $(\mathrm{h})$ & Yield (\%) \\
\hline $10 \mathrm{a}$ & $\mathrm{Me}, \mathrm{Me}$ & $n$-Pr & 24 & 87 \\
\hline $10 \mathrm{~b}$ & $\mathrm{Me}, \mathrm{Me}$ & $n-\mathrm{Bu}$ & 24 & 92 \\
\hline $10 \mathrm{c}$ & $\mathrm{Me}, \mathrm{Me}$ & $\mathrm{Bn}$ & 24 & 73 \\
\hline 10d & $\mathrm{Me}, \mathrm{Me}$ & 4- $\mathrm{ClC}_{6} \mathrm{H}_{4} \mathrm{CH}_{2}$ & 24 & 78 \\
\hline $10 \mathrm{e}$ & $\mathrm{Me}, \mathrm{Me}$ & $\mathrm{Ph}\left(\mathrm{CH}_{2}\right)_{2}$ & 24 & 86 \\
\hline $10 \mathrm{f}$ & $\mathrm{H}, \mathrm{H}$ & $n-\operatorname{Pr}$ & 24 & 86 \\
\hline $10 \mathrm{~g}$ & $\mathrm{H}, \mathrm{H}$ & $n-\mathrm{Bu}$ & 24 & 82 \\
\hline $10 \mathrm{~h}$ & $\mathrm{H}, \mathrm{H}$ & $\mathrm{Bn}$ & 24 & 85 \\
\hline $10 \mathrm{i}$ & $\mathrm{H}, \mathrm{H}$ & $4-\mathrm{ClC}_{6} \mathrm{H}_{4} \mathrm{CH}_{2}$ & 24 & 77 \\
\hline $10 \mathrm{j}$ & $\mathrm{H}, \mathrm{H}$ & $\mathrm{Ph}\left(\mathrm{CH}_{2}\right)_{2}$ & 24 & 72 \\
\hline $10 \mathrm{k}$ & $\mathrm{Br}, \mathrm{Br}$ & $n-\operatorname{Pr}$ & 36 & 98 \\
\hline 101 & $\mathrm{Br}, \mathrm{Br}$ & $n-\mathrm{Bu}$ & 36 & 82 \\
\hline $10 \mathrm{~m}$ & $\mathrm{Br}, \mathrm{Br}$ & $\mathrm{Bn}$ & 36 & 86 \\
\hline $10 n$ & $\mathrm{Br}, \mathrm{Br}$ & $4-\mathrm{ClC}_{6} \mathrm{H}_{4} \mathrm{CH}_{2}$ & 36 & 78 \\
\hline 100 & $\mathrm{Br}, \mathrm{Br}$ & $\mathrm{Ph}\left(\mathrm{CH}_{2}\right)_{2}$ & 36 & 79 \\
\hline $10 \mathrm{p}$ & $-\mathrm{HC}=\mathrm{CH}-\mathrm{CH}=\mathrm{CH}-$ & $n$-Pr & 24 & 85 \\
\hline $10 q$ & $-\mathrm{HC}=\mathrm{CH}-\mathrm{CH}=\mathrm{CH}-$ & $\mathrm{Bn}$ & 24 & 81 \\
\hline $10 r$ & $-\mathrm{HC}=\mathrm{CH}-\mathrm{CH}=\mathrm{CH}-$ & 4- $\mathrm{ClC}_{6} \mathrm{H}_{4} \mathrm{CH}_{2}$ & 24 & 75 \\
\hline
\end{tabular}

In summary, a library of hexahydropyrazino[1,2- $b]$ isoquinolines has been synthesised as representative simplified saframycin analogues. Both piperazinones and diketopiperazines were synthesised. The piperazinones

were further reacted with $\mathrm{LiAlH}_{4}$ to obtain piperazines or $\mathrm{LiAlH}(\mathrm{OEt})_{3}$ and $\mathrm{KCN}$ to insert an $\alpha$-aminonitrile function.

Table 2 Synthesis of Piperazinones 11<smiles>[H][R5](=N)OCC</smiles>

9a,b,d<smiles>[R7]N1CCN2Cc3c(c(OC)c([R][H])c([Tl])c3OC)CC2C1=O</smiles>

11a-k

\begin{tabular}{llll}
\hline Compd & $\mathrm{R}^{1}, \mathrm{R}^{1}$ & $\mathrm{R}^{2}$ & Yield (\%) \\
\hline 11a & $\mathrm{Me}, \mathrm{Me}$ & $n$-Pr & 75 \\
11b & $\mathrm{H}, \mathrm{H}$ & $n$-Pr & 79 \\
11c & $\mathrm{H}, \mathrm{H}$ & $n$-Bu & 70 \\
11d & $\mathrm{H}, \mathrm{H}$ & $\mathrm{Bn}$ & 59
\end{tabular}


Table 2 Synthesis of Piperazinones 11 (continued)

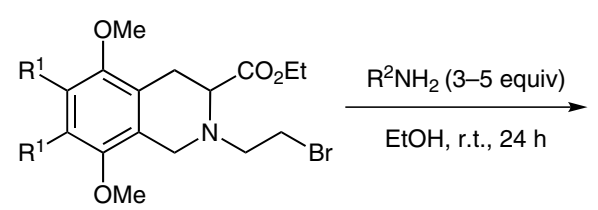

9a,b,d<smiles>[R7]c1c([R7])c(OC)c2c(c1OC)CC1C(=O)N([R])CCN1C2</smiles>

$11 a-k$

\begin{tabular}{|c|c|c|c|}
\hline Compd & $\mathrm{R}^{1}, \mathrm{R}^{1}$ & $\mathrm{R}^{2}$ & Yield (\%) \\
\hline $11 \mathrm{e}$ & $\mathrm{H}, \mathrm{H}$ & $4-\mathrm{ClC}_{6} \mathrm{H}_{4} \mathrm{CH}_{2}$ & 65 \\
\hline $11 f$ & $\mathrm{H}, \mathrm{H}$ & $\mathrm{Ph}\left(\mathrm{CH}_{2}\right)_{2}$ & 75 \\
\hline $11 \mathrm{~g}$ & $\mathrm{H}, \mathrm{H}$ & $4-\mathrm{ClC}_{6} \mathrm{H}_{4}\left(\mathrm{CH}_{2}\right)_{2}$ & 63 \\
\hline $11 \mathrm{~h}$ & $\mathrm{H}, \mathrm{H}$ & $2,5-(\mathrm{MeO})_{2} \mathrm{C}_{6} \mathrm{H}_{3}\left(\mathrm{CH}_{2}\right)_{2}$ & 74 \\
\hline $11 \mathbf{i}$ & $-\mathrm{HC}=\mathrm{CH}-\mathrm{CH}=\mathrm{CH}-$ & $n-\operatorname{Pr}$ & 72 \\
\hline $11 \mathrm{j}$ & $-\mathrm{HC}=\mathrm{CH}-\mathrm{CH}=\mathrm{CH}-$ & $n-\mathrm{Bu}$ & 70 \\
\hline $11 k$ & $-\mathrm{HC}=\mathrm{CH}-\mathrm{CH}=\mathrm{CH}-$ & $4-\mathrm{ClC}_{6} \mathrm{H}_{4} \mathrm{CH}_{2}$ & 75 \\
\hline
\end{tabular}

\section{Acknowledgment}

The authors are indebted to the Janssen Research Foundation (Johnson \& Johnson) for financial support.

Supporting Information for this article is available online at http://www.thieme-connect.com/ejournals/toc/synlett.

\section{References and Notes}

(1) Current address: Institute of Chemistry, Vietnam Academy of Science \& Technology, 18 Hoang Quoc Viet, Cau Giay, Ha Noi, Viet Nam.

(2) (a) Arai, T.; Takahashi, K.; Kubo, A. J. Antibiot. 1977, 30, 1015. (b) Arai, T.; Takahashi, K.; Ishiguro, K.; Yazawa, K. J. Antibiot. 1980, 33, 951. For a review on tetrahydroisoquinoline antitumor antibiotics, see: (c) Scott, J. D.; Williams, R. M. Chem. Rev. 2002, 102, 1669.

(3) (a) Wright, A. E.; Forleo, D. A.; Gunawardana, G. P.; Gunasekera, S. P.; Koehn, F. E.; McConnell, O. J. J. Org. Chem. 1990, 55, 4508. (b) Rinehart, K. T.; Holt, T. G.; Fregeau, N. L.; Stroh, J. G.; Keifer, P. A.; Sun, F.; Li, L. H.; Martin, D. G. J. Org. Chem. 1990, 55, 4512. (c) GarciaRocha, M.; Garcia-Gravalos, M. D.; Avila, J. Brit. J. Cancer 1996, 73, 875. (d) Schwartsmann, G.; Brondani da Rocha, A.; Berlinck, R. G. S.; Jimeno, J. Lancet Oncol. 2001, 2, 221. (e) Rinehart, K. L. Med. Res. Rev. 2000, 20, 1.

(4) Martinez, E. J.; Owa, T.; Schreiber, S. L.; Corey, E. J. Proc. Natl. Acad. Sci. U.S.A. 1999, 98, 3496.

(5) (a) Kubo, A.; Saito, N.; Yamato, H.; Masubuchi, K.; Nakamura, M. J. Org. Chem. 1988, 53, 4295. (b) Saito, N.; Harada, S.; Inouye, I.; Yamaguchi, K.; Kubo, A. Tetrahedron 1995, 51, 8231. (c) Obika, S.; Yasui, Y.; Yanada, R.; Takemoto, Y. J. Org. Chem. 2008, 73, 5206. (d) Martinez, E. J.; Corey, E. J. Org. Lett. 1999, 1, 75. (e) Dong, W.; Liu, W.; Liao, X.; Guan, B.; Chen, S.; Liu, Z. J. Org. Chem. 2011, 76, 5363.

(6) Huck, L.; González, J. F.; de la Cuesta, E.; Menéndeza, J. C.; Avendaño, C. Org. Biomol. Chem. 2011, 9, 6271.

(7) (a) González, J. F.; de la Cuesta, E.; Avendaño, C. Bioorg. Med. Chem. 2007, 15, 112. (b) Ortín, I.; González, J. C.; de la Cuesta, E.; Manguan-García, C.; Perona, R.; Avendaño,
C. Bioorg. Med. Chem. 2008, 16, 9065. (c) Ortín, I.; González, J. C.; de la Cuesta, E.; Avendaño, C. Bioorg. Med. Chem. 2010, 18, 6813.

(8) Nguyen Van, T.; Claes, P.; De Kimpe, N. Synlett 2013, 24, 1006.

(9) Mash, E. A.; Williams, L. J.; Pfeiffer, S. S. Tetrahedron Lett. 1997, 38, 6977.

(10) (a) Syper, L.; Mlochowski, J.; Kloc, K. Tetrahedron 1983 , 39, 781. (b) Ardecky, R. J.; Kerdesky, F. A. J.; Cava, M. P. J. Org. Chem. 1981, 46, 1483. (c) Eskildse, J.; Christensen, T.; Reenberg, T.; Larsen, U.; Christensen, J. B. Org. Prep. Proced. Int. 2000, 32, 398. (d) Mendez-Rojas, M. A.; Ejsmont, K.; Watson, W. H. J. Chem. Crystallogr. 2002, 32, 177. (e) Claessens, S.; Jacobs, J.; Van Aeken, S.; Abbaspour Tehrani, K.; De Kimpe, N. J. Org. Chem. 2008, 73, 7555.

(11) Ethyl 5,8-Dimethoxy-1,2,3,4-tetrahydroisoquinoline-3carboxylate (7b)

To a solution of ethyl $\mathrm{N}$-(diphenylmethylene)glycinate (4, $801 \mathrm{mg}, 3 \mathrm{mmol}$ ), freshly recrystallized 2,3-bisbromomethyl-1,4-dimethoxybenzene (5b, $972 \mathrm{mg}, 3 \mathrm{mmol}$ ), and $\mathrm{Bu}_{4} \mathrm{NHSO}_{4}(1017 \mathrm{mg}, 3 \mathrm{mmol})$ in $\mathrm{CH}_{2} \mathrm{Cl}_{2}(10 \mathrm{~mL})$ was added an aq solution of $\mathrm{KOH}(30 \%, 5 \mathrm{~mL})$. The reaction mixture was stirred at r.t. for $30 \mathrm{~min}$. Next, the mixture was poured onto $\mathrm{H}_{2} \mathrm{O}$ and exhaustively extracted with $\mathrm{CH}_{2} \mathrm{Cl}_{2}$. The combined organic phases were washed with sat. aq $\mathrm{NaHCO}_{3}$, dried $\left(\mathrm{MgSO}_{4}\right)$, filtered, and evaporated in vacuo. To this crude residue was added THF $(20 \mathrm{~mL})$ and $\mathrm{HCl}(2 \mathrm{M}$, $20 \mathrm{~mL}$ ). The reaction mixture was stirred at r.t. for $30 \mathrm{~min}$ and subsequently neutralized by treatment with a solution of aq $\mathrm{Na}_{2} \mathrm{CO}_{3}(2 \mathrm{M})$. Next, the solvent was evaporated in vacuo, and the residue was exhaustively extracted with EtOAc. The combined organic phases were washed with sat. aq $\mathrm{NaHCO}_{3}$, dried $\left(\mathrm{MgSO}_{4}\right)$, filtered, and evaporated in vacuo. This crude mixture was purified column chromatography on silica (hexane-EtOAc) to obtain ethyl 1,2,3,4-tetrahydroisoquinoline-3-carboxylate $(7 \mathbf{b}, 637 \mathrm{mg}$, $80 \%$ ).

Note: In order to obtain a good yield, it is of utmost importance to use freshly recrystallized starting materials $\mathbf{4}$ and 5.

Analytical Data

Colourless crystals; mp 90.5-91 ${ }^{\circ} \mathrm{C} .{ }^{1} \mathrm{H}$ NMR $(270 \mathrm{MHz}$, $\left.\mathrm{CDCl}_{3}\right): \delta=1.31(3 \mathrm{H}, \mathrm{t}, J=7.0 \mathrm{~Hz}, \mathrm{Me}), 1.63(1 \mathrm{H}, \mathrm{br} \mathrm{s}$, 
NH), $2.64(1 \mathrm{H}, \mathrm{dd}, J=9.9,16.5 \mathrm{~Hz}, \mathrm{H}-4 \mathrm{a}), 3.09(1 \mathrm{H}, \mathrm{dd}$, $J=16.5,4.6 \mathrm{~Hz}, \mathrm{H}-4 \mathrm{~b}), 3.59$ (1 H, dd, $J=4.6,9.9 \mathrm{~Hz}, \mathrm{H}-3)$, $3.76(3 \mathrm{H}, \mathrm{s}, \mathrm{OMe}), 3.78(3 \mathrm{H}, \mathrm{s}, \mathrm{OMe}), 3.80(1 \mathrm{H}, \mathrm{d}, J=15.8$ $\mathrm{Hz}, \mathrm{H}-1 \mathrm{a}), 3.84(1 \mathrm{H}, \mathrm{d}, J=15.8 \mathrm{~Hz}, \mathrm{H}-1 \mathrm{~b}), 4.19-4.27(2 \mathrm{H}$, $\left.\mathrm{m}, \mathrm{OCH}_{2}\right), 6.60(1 \mathrm{H}, \mathrm{d}, J=8.9 \mathrm{~Hz}, \mathrm{H}-6) 6.64(1 \mathrm{H}, \mathrm{d}, J=8.9$ $\mathrm{Hz}, \mathrm{H}-7) .{ }^{13} \mathrm{C}$ NMR $\left(68 \mathrm{MHz}, \mathrm{CDCl}_{3}\right): \delta=14.2(\mathrm{Me}), 26.3$ (C-4), 42.9 (C-1), 55.3 (C-3), 55.4 (OMe), 55.6 (OMe), 60.9 $\left(\mathrm{OCH}_{2}\right), 106.9$ and $107.2(\mathrm{C}-6, \mathrm{C}-7), 123.7$ and 125.1 (C-5a, C-8a), $149.9(=\mathrm{COMe}), 151.2(=\mathrm{COMe}), 173.3(\mathrm{C}=\mathrm{O})$. IR $(\mathrm{KBr}): v=3250(\mathrm{NH}), 2971,2954,2829,1724(\mathrm{C}=\mathrm{O}), 1605$, 1484, 1464, 1438, 1260, 1225, 1182, $1091 \mathrm{~cm}^{-1}$. MS: $\mathrm{m} / \mathrm{z}$ $(\%)=266(100)\left[\mathrm{M}+\mathrm{H}^{+}\right], 262(20), 261(20), 192(20)$. HRMS $\left(\mathrm{ES}^{+}\right): \mathrm{m} / z$ calcd for $\left[\mathrm{C}_{16} \mathrm{H}_{22} \mathrm{NO}_{4}\right]^{+}: 266.1392$; found: 266.1396. Spectroscopic data are in accordance with literature data: Al-Horani, R. A.; Desai, U. R. Tetrahedron 2012, 68, 2027.

(12) Ethyl 2-(2-Chloroacetyl)-5,8-dimethoxy-6,7-dimethyl1,2,3,4-tetrahydroisoquinoline-3-carboxylate (8a) A mixture of ethyl 5,8-dimethoxy-6,7-dimethyl-1,2,3,4tetrahydroisoquinoline-3-carboxylate (7a, $586 \mathrm{mg}, 2 \mathrm{mmol}$ ) and $\mathrm{Et}_{3} \mathrm{~N}(222 \mathrm{mg}, 2.2 \mathrm{mmol})$ in anhydrous $\mathrm{CH}_{2} \mathrm{Cl}_{2}(10 \mathrm{~mL})$ was cooled to $0{ }^{\circ} \mathrm{C}$ and chloroacetyl chloride $(264 \mathrm{mg}, 2.2$ mmol) was added dropwise. The reaction mixture was stirred at r.t. for $2 \mathrm{~h}$. Then the mixture was poured onto $\mathrm{H}_{2} \mathrm{O}$ and exhaustively extracted with $\mathrm{CH}_{2} \mathrm{Cl}_{2}$. The combined organic phases were washed with sat. $\mathrm{NaHCO}_{3}$, dried $\left(\mathrm{MgSO}_{4}\right)$, filtered, and evaporated in vacuo. Purification by column chromatography on silica (hexane-EtOAc) gave pure ethyl 2-(2-chloroacetyl)-5,8-dimethoxy-6,7-dimethyl1,2,3,4-tetrahydroisoquinoline-3-carboxylate (8a, $688 \mathrm{mg}$, $93 \%)$.

\section{Analytical Data}

${ }^{1} \mathrm{H}$ NMR $\left(300 \mathrm{MHz}, \mathrm{CDCl}_{3}\right): \delta=1.15(3 \mathrm{H}, \mathrm{t}, J=6.9 \mathrm{~Hz}$, $\mathrm{Me}), 2.17(6 \mathrm{H}, \mathrm{s}, 2 \times \mathrm{Me}), 2.90(1 \mathrm{H}, \mathrm{dd}, J=5.9,16.5 \mathrm{~Hz}$, H-4a), 3.47 ( $1 \mathrm{H}, \mathrm{dd}, J=3.0,16.5 \mathrm{~Hz}, \mathrm{H}-4 \mathrm{~b}), 3.65(3 \mathrm{H}, \mathrm{s}$, $\mathrm{OMe}), 3.68$ (3 H, s, OMe), 4.00-4.17 (2 H, m, $\left.\mathrm{OCH}_{2}\right), 4.24$ $(1 \mathrm{H}, \mathrm{d}, J=12.5 \mathrm{~Hz}, \mathrm{H}-1 \mathrm{a}), 4.29(1 \mathrm{H}, \mathrm{d}, J=12.5 \mathrm{~Hz}, \mathrm{H}-1 \mathrm{~b})$, $4.73\left(2 \mathrm{H}\right.$, br s, $\left.2 \times \mathrm{H}^{-2} 2^{\prime}\right), 5.46(1 \mathrm{H}, \mathrm{dd}, J=3.0,5.9 \mathrm{~Hz}, \mathrm{H}-$ 3). ${ }^{13} \mathrm{C} \mathrm{NMR}\left(75 \mathrm{MHz}, \mathrm{CDCl}_{3}\right): \delta=12.51(\mathrm{Me}), 12.54(\mathrm{Me})$, $14.0\left(\mathrm{OCH}_{2} \mathrm{Me}\right), 24.8(\mathrm{C}-4), 41.2\left(\mathrm{C}-2^{\prime}\right), 41.4(\mathrm{C}-1), 51.3(\mathrm{C}-$ 3), $60.4(2 \times \mathrm{OMe}), 61.4\left(\mathrm{OCH}_{2}\right), 122.6$ and $123.2(\mathrm{C}-5 \mathrm{a}, \mathrm{C}-$ 8a), 129.2 and 129.8 (C-6, C-7), $150.6(=\mathrm{COMe}), 152.0$ $(=\mathrm{COMe}), 166.6$ and $170.3(2 \times \mathrm{C}=\mathrm{O})$. IR $(\mathrm{NaCl}): v=2942$, 2838, $1738(\mathrm{C}=\mathrm{O}), 1732(\mathrm{C}=\mathrm{O}), 1660,1652,1606,1486$, 1483, 1260, 1203, $1096 \mathrm{~cm}^{-1}$. MS: $\mathrm{m} / z(\%)=370 / 372(100)$ [M + $\left.\mathrm{H}^{+}\right], 324$ (20), 296 (55), 294 (80), 266 (30), 220 (10). HRMS (ES ${ }^{+}$: $m / z$ calcd for $\left[\mathrm{C}_{18} \mathrm{H}_{25}{ }^{35} \mathrm{ClNO}_{5}\right]^{+}: 372.1392$; found: 372.1401 .

(13) Ethyl 2-(2-Bromoethyl)-5,8-dimethoxy-1,2,3,4tetrahydroisoquinoline-3-carboxylate (9b) A mixture of tetrahydroisoquinoline $7 \mathbf{b}(800 \mathrm{mg}, 3.02$ $\mathrm{mmol}), 1,2$-dibromoethane $(11.35 \mathrm{~g}, 60.4 \mathrm{mmol})$, and $\mathrm{K}_{2} \mathrm{CO}_{3}(417 \mathrm{mg}, 3.02 \mathrm{mmol})$ was stirred at reflux for $24 \mathrm{~h}$. Then, the mixture was poured onto $\mathrm{H}_{2} \mathrm{O}$ and exhaustively extracted with EtOAc. The combined organic phases were washed with sat. $\mathrm{NaHCO}_{3}$, dried $\left(\mathrm{MgSO}_{4}\right)$, filtered, and evaporated in vacuo. Purification chromatography on silica (hexane-EtOAc) gave pure ethyl 2-(2-bromoethyl)-5,8dimethoxy-1,2,3,4-tetrahydroisoquinoline-3-carboxylate (9b, $786 \mathrm{mg}, 70 \%$ ).

Analytical Data

${ }^{1} \mathrm{H}$ NMR $\left(300 \mathrm{MHz}, \mathrm{CDCl}_{3}\right): \delta=1.23(3 \mathrm{H}, \mathrm{t}, J=7.0 \mathrm{~Hz}$, Me), $2.95(1 \mathrm{H}, \mathrm{dd}, J=6.3,17.3 \mathrm{~Hz}, \mathrm{H}-4 \mathrm{a}), 3.08-3.18(2 \mathrm{H}$, m, $\left.\mathrm{CH}_{2}-1^{\prime}\right), 3.20(1 \mathrm{H}, \mathrm{dd}, J=6.1,17.3 \mathrm{~Hz}, \mathrm{H}-4 \mathrm{~b}), 3.50(2 \mathrm{H}$, $\left.\mathrm{t}, J=7.2 \mathrm{~Hz}, \mathrm{CH}_{2}-2^{\prime}\right), 3.75(3 \mathrm{H}, \mathrm{s}, \mathrm{OMe}), 3.76(3 \mathrm{H}, \mathrm{s}$, OMe), 3.76 (1 H, dd, overlap, H-3), 3.85 (1 H, d, $J=16.8$ $\mathrm{Hz}, \mathrm{H}-1 \mathrm{a}), 3.96$ (1 H,d, $J=16.8 \mathrm{~Hz}, \mathrm{H}-1 \mathrm{~b}), 4.07-4.18$ (2 H, m, $\left.\mathrm{OCH}_{2}\right), 6.60(1 \mathrm{H}, \mathrm{d}, J=9.1 \mathrm{~Hz}, \mathrm{H}-6), 6.62(1 \mathrm{H}, \mathrm{d}$, $J=9.1 \mathrm{~Hz}, \mathrm{H}-7) .{ }^{13} \mathrm{C} \mathrm{NMR}\left(75 \mathrm{MHz}, \mathrm{CDCl}_{3}\right): \delta=14.4(\mathrm{Me})$, $25.8(\mathrm{C}-4), 30.3\left(\mathrm{C}-1^{\prime}\right), 46.9(\mathrm{C}-1), 55.5(\mathrm{OMe}), 55.8(\mathrm{OMe})$, $57.1(\mathrm{CBr}), 59.7(\mathrm{C}-3), 60.7\left(\mathrm{OCH}_{2}\right), 107.0$ and $107.4(\mathrm{C}-6$, $\mathrm{C}-7), 122.6$ and 123.8 (C-5a, C-8a), 150.1 (=COMe), 151.2 $(=\mathrm{COMe}), 172.5(\mathrm{C}=\mathrm{O})$. IR $(\mathrm{NaCl}): v=2930,1731(\mathrm{C}=\mathrm{O})$, $1650,1483,1464,1438,1257,1181,1082 \mathrm{~cm}^{-1}$. MS m/z (\%) 372/374 (M+H' 10$), 310$ (7), 393 (15), 392 (100). HRMS $\left(\mathrm{ES}^{+}\right): \mathrm{m} / z$ calcd for $\left[\mathrm{C}_{20} \mathrm{H}_{14} \mathrm{NO}_{2}\right]^{+}: 300.1025$; found: 300.1027 .

(14) 7,10-Dimethoxy-8,9-dimethyl-2-propyl-2,3,11,11atetrahydro- $6 \mathrm{H}$-pyrazino[1,2-b]isoquinoline-1,4-dione (10a)

A mixture of ethyl 2-(2-chloroacetyl)-5,8-dimethoxy-6,7dimethyl-1,2,3,4-tetrahydroisoquinoline-3-carboxylate (8a, $184.5 \mathrm{mg}, 0.5 \mathrm{mmol}$ ) and $n$-propylamine (132.5 mg, 2.5 $\mathrm{mmol})$ in anhydrous $\mathrm{EtOH}(10 \mathrm{~mL})$ was stirred for $24 \mathrm{~h}$ at r.t. Then, the mixture was poured onto $\mathrm{H}_{2} \mathrm{O}$ and exhaustively extracted with EtOAc. The combined organic phases were washed, dried $\left(\mathrm{MgSO}_{4}\right)$, filtered, and evaporated in vacuo. Purification by chromatography on silica (hexane-EtOAc) gave pure 7,10-dimethoxy-8,9-dimethyl-2-propyl-

2,3,11,11a-tetrahydro- $6 H$-pyrazino[1,2-b]isoquinoline-1,4dione (10a, $150 \mathrm{mg}, 87 \%$ ).

Analytical Data

White powder, mp $169-169.5^{\circ} \mathrm{C} .{ }^{1} \mathrm{H}$ NMR $(270 \mathrm{MHz}$, $\left.\mathrm{CDCl}_{3}\right): \delta=0.96(3 \mathrm{H}, \mathrm{t}, J=7.3 \mathrm{~Hz}, \mathrm{H}-3), 1.57-1.68(2 \mathrm{H}$, $\left.\mathrm{m}, 2 \times \mathrm{H}-2^{\prime}\right), 2.18(6 \mathrm{H}, \mathrm{s}, 2 \times \mathrm{Me}), 2.74(1 \mathrm{H}, \mathrm{dd}, J=12.2$, 16.4 Hz, H-11a), 3.28-3.47 (2 H, m, H-1'a, H-1'b), 3.59 (1 $\mathrm{H}, \mathrm{dd}, J=3.4,16.4 \mathrm{~Hz}, \mathrm{H}-11 \mathrm{~b}$ ), 3.66 (3 H, s, OMe), 3.71 (3 $\mathrm{H}, \mathrm{s}, \mathrm{OMe}), 4.04$ (2 H, s, H-3a, H-3b), 4.10 (1 H, d, $J=17.5$ $\mathrm{Hz}, \mathrm{H}-6 \mathrm{a}), 4.17$ (1 H, dd, $J=12.2,3.6 \mathrm{~Hz}, \mathrm{H}-12), 5.40(1 \mathrm{H}$, $\mathrm{d}, J=17.5 \mathrm{~Hz}, \mathrm{H}-6 \mathrm{~b}) .{ }^{13} \mathrm{C}$ NMR $\left(68 \mathrm{MHz}, \mathrm{CDCl}_{3}\right): \delta=11.1$ (C-3'), $12.45(\mathrm{Me}), 12.54(\mathrm{Me}), 19.8\left(\mathrm{C}-2^{\prime}\right), 28.5(\mathrm{C}-11)$, 40.1 (C-6), $47.6\left(\mathrm{C}-1^{\prime}\right), 49.4$ (C-3), 55.6 (C-12), 60.3 (2 × OMe), 122.9 and 123.9 (C-7a, C-10a), 129.3 and 129.5 (C8, C-9), 151.0 (=COMe), 152.1 (=COMe), $162.3(\mathrm{C}=\mathrm{O})$, $165.0(\mathrm{C}=\mathrm{O})$. IR $(\mathrm{KBr}): v=2958,2834,1661(\mathrm{C}=\mathrm{O}), 1658$ $(\mathrm{C}=\mathrm{O}), 1479,1465,1334,1260,1274,1086,1061 \mathrm{~cm}^{-1}$. MS: $m / z(\%)=347(30)[\mathrm{M}+\mathrm{H}], 345(70), 314(15), 218(35)$, 191 (100), 176 (70), 124 (50), 83 (70). HRMS (ES $): m / z$ calcd for $\left[\mathrm{C}_{19} \mathrm{H}_{27} \mathrm{~N}_{2} \mathrm{O}_{4}\right]^{+}: 347.1971$; found: 347.1981 .

(15) 7,10-Dimethoxy-2-propyl-3,4,11,11a-tetrahydro- $2 \mathrm{H,6H}$ pyrazino[1,2-b]isoquinolin-1-one (11b)

A mixture of ethyl 2-(2-bromoethyl)-5,8-dimethoxy-1,2,3,4tetrahydroisoquinoline-3-carboxylate (9b) $(186 \mathrm{mg}, 0.5$ $\mathrm{mmol})$ and $n$-propylamine $(132.5 \mathrm{mg}, 2.5 \mathrm{mmol})$ in anhydrous EtOH $(10 \mathrm{~mL})$ was stirred for $24 \mathrm{~h}$ at r.t. Then, the mixture was poured onto $\mathrm{H}_{2} \mathrm{O}$ and exhaustively extracted with EtOAc. The combined organic phases were washed, dried $\left(\mathrm{MgSO}_{4}\right)$, filtered, and evaporated in vacuo.

Purification by chromatography on silica (hexane-EtOAc) gave pure 7,10-dimethoxy-2-propyl-3,4,11,11a-tetrahydro$2 \mathrm{H}, 6 \mathrm{H}$-pyrazino[1,2-b]isoquinolin-1-one (11b, $120 \mathrm{mg}$, $79 \%)$.

\section{Analytical Data}

${ }^{1} \mathrm{H} \mathrm{NMR}\left(270 \mathrm{MHz}, \mathrm{CDCl}_{3}\right): \delta=0.91(3 \mathrm{H}, \mathrm{t}, J=7.3 \mathrm{~Hz}$, $\mathrm{Me}), 1.55-1.64\left(2 \mathrm{H}, \mathrm{m}, \mathrm{CH}_{2}-2^{\prime}\right), 2.59-2.69(2 \mathrm{H}, \mathrm{m}, \mathrm{H}-11 \mathrm{la}$, H-3a), 2.96 ( $1 \mathrm{H}, \mathrm{dd}, J=3.9,11.5 \mathrm{~Hz}, \mathrm{H}-12$ ), 3.09-3.29 (4 H, m, H-3b, H-4a, H-6a, H-1'a), 3.41-3.70 (3 H, m, H-4b, H1'b, H-11b), 3.74 (3 H, s, OMe), 3.76 (3 H, s, OMe), 4.10 (1 $\mathrm{H}, \mathrm{d}, J=15.5 \mathrm{~Hz}, \mathrm{H}-6 \mathrm{~b}), 6.61(1 \mathrm{H}, \mathrm{d}, J=8.1 \mathrm{~Hz}, \mathrm{H}-8), 6.63$ $(1 \mathrm{H}, \mathrm{d}, J=8.1 \mathrm{~Hz}, \mathrm{H}-9) \cdot{ }^{13} \mathrm{C} \mathrm{NMR}\left(68 \mathrm{MHz}, \mathrm{CDCl}_{3}\right): \delta=$ 11.2 (Me), 20.2 (C-2'), 27.6 (C-11), 46.1 (C-4), $48.4\left(\mathrm{C}-1^{\prime}\right)$, 50.4 (C-3), $53.2(\mathrm{C}-6), 55.6(\mathrm{OMe}), 55.7(\mathrm{OMe}), 61.5(\mathrm{C}-$ 12), 107.1 and 107.7 (C-8, C-9), 123.4 and 124.7 (C-7a, C10a), 149.7 (=COMe), 151.5 (=COMe), $168.6(\mathrm{C}=\mathrm{O})$. IR 
$(\mathrm{NaCl}): v=2931,1654(\mathrm{C}=\mathrm{O}), 1645,1485,1463,1438$, 1259, 1181, $1097 \mathrm{~cm}^{-1}$. MS: $m / z(\%)=305(100)\left[\mathrm{M}+\mathrm{H}^{+}\right]$, 301 (10), 227 (20). HRMS (ES $)^{+}: \mathrm{m} / z$ calcd for $\left[\mathrm{C}_{17} \mathrm{H}_{25} \mathrm{~N}_{2} \mathrm{O}_{3}\right]^{+}: 305.1865$; found: 305.1871 .

(16) 7,10-Dimethoxy-2-propyl-1,3,4,6,11,11a-hexahydro-2Hpyrazino[1,2-b]isoquinoline (14a)

To a solution of 7,10-dimethoxy-2-propyl-3,4,11,11atetrahydro- $2 H, 6 H$-pyrazino[1,2-b]isoquinolin-1-one (11b, $100 \mathrm{mg}, 0.33 \mathrm{mmol})$ in anhydrous $\mathrm{Et}_{2} \mathrm{O}(5 \mathrm{~mL})$ under a nitrogen atmosphere at $0{ }^{\circ} \mathrm{C}$, was added $\mathrm{LiAlH}_{4}(53 \mathrm{mg}, 1.32$ $\mathrm{mmol}$ ) portionwise. The reaction mixture was stirred for 12 $\mathrm{h}$ at r.t. Afterwards, the mixture was poured onto $\mathrm{H}_{2} \mathrm{O}$ and exhaustively extracted with $\mathrm{Et}_{2} \mathrm{O}$. The combined organic phases were washed, dried $\left(\mathrm{MgSO}_{4}\right)$, filtered, and evaporated in vacuo. Purification by chromatography on silica (hexane-EtOAc) gave pure 7,10-dimethoxy-2-propyl$1,3,4,6,11,11 a$-hexahydro- $2 H$-pyrazino $[1,2-b]$ isoquinoline (14a, $76 \mathrm{mg}, 80 \%$ ).

Analytical Data

Pale white solid; $\mathrm{mp} 105^{\circ} \mathrm{C} .{ }^{1} \mathrm{H}$ NMR $\left(270 \mathrm{MHz}, \mathrm{CDCl}_{3}\right): \delta$ $=0.92\left(3 \mathrm{H}, \mathrm{t}, J=7.3 \mathrm{~Hz}, \mathrm{CH}_{3}\right), 1.50-1.59\left(2 \mathrm{H}, \mathrm{m}, \mathrm{CH}_{2}-2^{\prime}\right)$, $1.93(1 \mathrm{H}, \mathrm{dd}, J=9.7,11.0 \mathrm{~Hz}, \mathrm{H}-1 \mathrm{a}), 2.21-2.36(3 \mathrm{H}, \mathrm{m}$, overlap, H-3a, H-1'a, H-1'b), 2.41-2.53 (2 H, m, overlap, H11a, H-4a), $2.79(1 \mathrm{H}, \mathrm{d}, J=14.2 \mathrm{~Hz}, \mathrm{H}-11 \mathrm{~b}), 2.95(1 \mathrm{H}, \mathrm{dd}$, $J=2.3,11 \mathrm{~Hz}, \mathrm{H}-1 \mathrm{~b}), 3.01-3.09$ (2 H, m, H-3b, H-4b), 3.13 $(1 \mathrm{H}, \mathrm{d}, J=16.0 \mathrm{~Hz}, \mathrm{H}-6 \mathrm{a}), 3.77(6 \mathrm{H}, \mathrm{s}, 2 \times \mathrm{OMe}), 4.06(1$ $\mathrm{H}, \mathrm{d}, J=16.0 \mathrm{~Hz}, \mathrm{H}-6 \mathrm{~b}), 6.62(2 \mathrm{H}, \mathrm{s}, \mathrm{H}-9, \mathrm{H}-8) .{ }^{13} \mathrm{C}$ NMR $\left(68 \mathrm{MHz}, \mathrm{CDCl}_{3}\right): \delta=12.0(\mathrm{Me}), 20.0\left(\mathrm{C}-2^{\prime}\right), 28.2(\mathrm{C}-11)$, $52.1(\mathrm{C}-6), 53.2(\mathrm{C}-4), 54.6(\mathrm{C}-3), 55.6(2 \times \mathrm{OMe}), 60.1(\mathrm{C}-$ 1'), 60.7 (C-1), 76.6 (C-11a), 107.0 and 107.2 (C-8, C-9), 123.6 and $124.2(\mathrm{C} 7 \mathrm{a}, \mathrm{C}-10 \mathrm{a}), 149.8(=\mathrm{COMe}), 150.9$ $(=\mathrm{COMe})$. IR (ATR): $v=2925,1654,1482,1438,1258$, 1086, 1060, 810, $714 \mathrm{~cm}^{-1}$. MS: $m / z(\%)=291(100)[\mathrm{M}+$ $\left.\mathrm{H}^{+}\right]$. HRMS $\left(\mathrm{ES}^{+}\right): \mathrm{m} / \mathrm{z}$ calcd for $\left[\mathrm{C}_{17} \mathrm{H}_{27} \mathrm{~N}_{2} \mathrm{O}_{2}\right]^{+}: 291.2073$; found: 291.2065 .

(17) 7,10-Dimethoxy-2-propyl-1,2,3,4,6,11-hexahydropyrazino[1,2-b]isoquinoline-11a-carbonitrile (13a) To a mixture of fresh $\mathrm{LiAlH}_{4}(40 \mathrm{mg}, 1.0 \mathrm{mmol})$ in anhydrous $\mathrm{Et}_{2} \mathrm{O}(5 \mathrm{~mL})$ was added anhydrous $\mathrm{EtOH}(0.175$ $\mathrm{mL}, 3.0 \mathrm{mmol}$ ) under a nitrogen atmosphere at $0{ }^{\circ} \mathrm{C}$. After $90 \mathrm{~min}$, a solution of $11 \mathrm{a}(30 \mathrm{mg}, 0.1 \mathrm{mmol})$ in anhydrous THF $(5 \mathrm{~mL})$ was added, and the reaction mixture was stirred at $0{ }^{\circ} \mathrm{C}$ for $30-50 \mathrm{~min}$. Next, $\mathrm{AcOH}(0.226 \mathrm{~mL}, 4 \mathrm{mmol})$ was added. After $5 \mathrm{~min}, \mathrm{KCN}$ (40 mg, $0.61 \mathrm{mmol}$ ) in $\mathrm{H}_{2} \mathrm{O}$ was added dropwise (CAUTION: HCN formation!). The reaction mixture was stirred for $5 \mathrm{~h}$ at r.t., then the mixture was poured onto $\mathrm{H}_{2} \mathrm{O}$, neutralised with sat. aq $\mathrm{NaHCO}_{3}$ solution and exhaustively extracted with EtOAc. The combined organic phases were washed, dried $\left(\mathrm{MgSO}_{4}\right)$, filtered, and evaporated in vacuo. Purification by chromatography on silica (hexane-EtOAc) gave pure 7,10- dimethoxy-2-propyl-1,2,3,4,6,11-hexahydropyrazino[1,2$b$ ]isoquinoline-11a-carbonitrile (13a, $26 \mathrm{mg}, 84 \%$ ).

\section{Analytical Data}

White powder; mp $128.5-129.3^{\circ} \mathrm{C} .{ }^{1} \mathrm{H}$ NMR $(300 \mathrm{MHz}$, $\left.\mathrm{CDCl}_{3}\right): \delta=0.94\left(3 \mathrm{H}, \mathrm{t}, J=7.3 \mathrm{~Hz}, \mathrm{CH}_{3}-3\right), 1.52-1.57(2 \mathrm{H}$, $\left.\mathrm{m}, 2 \times \mathrm{H}-2^{\prime}\right), 2.11(1 \mathrm{H}, \mathrm{d}, J=11.3 \mathrm{~Hz}, \mathrm{H}-1 \mathrm{a}), 2.27-2.38$ (3 H, m, overlap, H-3a, H-1'a, H-1'b), 2.63-2.71 (2 H, m, overlap, H-11 a, H-4a), 2.84-2.91 (2 H, m, overlap, H-3b, H4b), $3.12(1 \mathrm{H}, \mathrm{d}, J=17.0 \mathrm{~Hz}, \mathrm{H}-11 \mathrm{~b}), 3.21(1 \mathrm{H}, \mathrm{d}, J=11.3$ $\mathrm{Hz}, \mathrm{H}-1 \mathrm{~b}), 3.28(1 \mathrm{H}, \mathrm{d}, J=16.8 \mathrm{~Hz}, \mathrm{H}-6 \mathrm{a}), 3.76(3 \mathrm{H}, \mathrm{s}$, OMe), 3.78 (3 H, s, OMe), $4.00(1 \mathrm{H}, J=16.8 \mathrm{~Hz}, \mathrm{H}-6 \mathrm{~b})$, $6.63(2 \mathrm{H}, \mathrm{s}, \mathrm{H}-9, \mathrm{H}-8) .{ }^{13} \mathrm{C} \mathrm{NMR}\left(75 \mathrm{MHz}, \mathrm{CDCl}_{3}\right): \delta=11.7$ (Me), 19.8 (C-2'), 32.3 (C-11), 49.1 (C-6), 51.5 (C-4), 52.7 (C-3), $55.5(2 \times \mathrm{OMe}), 56.7(\mathrm{C}-11 \mathrm{a}), 59.5\left(\mathrm{C}-1^{\prime}\right), 61.7(\mathrm{C}-1)$, 107.5 and $107.6(\mathrm{C}-7, \mathrm{C}-8), 117.5(\mathrm{CN}), 119.9$ and 122.7 (C7a, C-10a), 149.6 (=COMe), 150.7 (=COMe). IR (KBr): v $=2931,2835,2183(\mathrm{CN}), 1652,1607,1486,1463,1456$, $1259,1172,1080 \mathrm{~cm}^{-1}$. MS: $m / z(\%)=316(15)\left[\mathrm{M}+\mathrm{H}^{+}\right]$, 301 (15), 290 (25), 289 (100). HRMS (ES $\left.{ }^{+}\right): \mathrm{m} / z$ calcd for $\left[\mathrm{C}_{18} \mathrm{H}_{26} \mathrm{~N}_{3} \mathrm{O}_{2}\right]^{+}: 316.2025$; found: 316.2025 .

(18) 2-Benzyl-3,4,11,11a-tetrahydro-2 $H, 6 H$-pyrazino[1,2b] isoquinoline-1,7,10-trione (12)

To a solution of 7,10-dimethoxy-2-benzyl-3,4,11,11atetrahydro-2H,6H-pyrazino[1,2-b]isoquinolin-1-one (11d, $176 \mathrm{mg}, 0.5 \mathrm{mmol})$ in anhydrous $\mathrm{CH}_{2} \mathrm{Cl}_{2}(20 \mathrm{~mL})$ was added dropwise $\mathrm{BBr}_{3}(1035 \mathrm{mg}, 1.05 \mathrm{mmol})$ under a nitrogen atmosphere at $-78^{\circ} \mathrm{C}$. After $1 \mathrm{~h}$, the reaction mixture was warmed to $0{ }^{\circ} \mathrm{C}$ and left for $30 \mathrm{~min}$. Then, $\mathrm{HNO}_{3}(10 \mathrm{M}$, $10 \mathrm{~mL}$ ) was added to the reaction mixture and stirring was continued for $45 \mathrm{~min}$. Next, the mixture was poured onto $\mathrm{H}_{2} \mathrm{O}$, neutralised with a sat. aq $\mathrm{NaHCO}_{3}$ solution and exhaustively extracted with $\mathrm{CH}_{2} \mathrm{Cl}_{2}$. The combined organic phases were washed, dried $\left(\mathrm{MgSO}_{4}\right)$, filtered, and evaporated in vacuo. Purification by chromatography on silica (hexane-EtOAc) gave pure 2-benzyl-3,4,11,11atetrahydro- $2 H, 6 H$-pyrazino[1,2- $b$ ]isoquinoline-1,7,10trione (12, $110 \mathrm{mg}, 68 \%)$.

\section{Analytical Data}

${ }^{1} \mathrm{H} \mathrm{NMR}\left(270 \mathrm{MHz}, \mathrm{CDCl}_{3}\right): \delta=2.47-2.60(1 \mathrm{H}, \mathrm{m}, \mathrm{H}-11 \mathrm{a})$, $2.64(1 \mathrm{H}, \mathrm{dt}, J=3.6,12.3 \mathrm{~Hz}, \mathrm{H}-3 \mathrm{a}), 3.02(1 \mathrm{H}, \mathrm{dd}, J=4.1$, $10.6 \mathrm{~Hz}, \mathrm{H}-12$ ), 3.06-3.19 (3 H, m, overlap, H-4a, H-3b, H6a), 3.33 (1 H, td, $J=3.6,19.8 \mathrm{~Hz}, \mathrm{H}-4 \mathrm{~b}), 3.49(1 \mathrm{H}, \mathrm{dt}$, $J=4.1,11.2 \mathrm{~Hz}, \mathrm{H}-11 \mathrm{~b}), 3.90(1 \mathrm{H}, \mathrm{dd}, J=1.3,19.8 \mathrm{~Hz}, \mathrm{H}-$ 6b), $4.53\left(1 \mathrm{H}, \mathrm{d}, J=14.5 \mathrm{~Hz}, \mathrm{H}-1^{\prime} \mathrm{a}\right), 4.73(1 \mathrm{H}, \mathrm{d}, J=14.5$ $\left.\mathrm{Hz}, \mathrm{H}-1^{\prime} \mathrm{b}\right), 6.71(1 \mathrm{H}, \mathrm{d}, J=10.2 \mathrm{~Hz}, \mathrm{H}-8), 6.76(1 \mathrm{H}, \mathrm{d}$, $J=10.2 \mathrm{~Hz}, \mathrm{H}-9), 7.26-7.37(5 \mathrm{H}, \mathrm{m}, 5 \times=\mathrm{CH}) .{ }^{13} \mathrm{C} \mathrm{NMR}$ $\left(68 \mathrm{MHz}, \mathrm{CDCl}_{3}\right): \delta=26.4(\mathrm{C}-11), 45.2(\mathrm{C}-4), 49.8(\mathrm{C}-3)$, 49.9 (C-1'), 51.5 (C-6), $60.4(\mathrm{C}-12), 127.7(=\mathrm{CH}), 128.1$ $(2 \times=\mathrm{CH}), 128.7(2 \times=\mathrm{CH}), 136.0(\mathrm{C}-8), 136.3\left(\mathrm{C}_{\text {quat }}\right)$, $136.5(\mathrm{C}-9), 138.6\left(\mathrm{C}_{\text {quat }}\right), 140.4\left(\mathrm{C}_{\text {quat }}\right), 167.4(\mathrm{C}=\mathrm{O}), 185.7$ $(\mathrm{C}=\mathrm{O}), 185.9(\mathrm{C}=\mathrm{O})$. IR $(\mathrm{NaCl}): v=2924,1660(\mathrm{C}=\mathrm{O})$, $1641(\mathrm{C}=\mathrm{O}), 1496,1453,1352,1311,1250 \mathrm{~cm}^{-1}$. MS: $\mathrm{m} / \mathrm{z}$ $(\%)=323(5)\left[\mathrm{M}+\mathrm{H}^{+}\right], 322(20), 321(100), 178(7)$. 